Trauma Berufskrankh $2013 \cdot 15$ [Suppl 1]:59-66 DOI 10.1007/s10039-012-1916-3

Online publiziert: 30. Januar 2013

c) Springer-Verlag Berlin Heidelberg 2013
D.W. Sommerfeldt

Abteilung für Kinder- und Jugendtraumatologie, Altonaer Kinderkrankenhaus gGmbH, Hamburg

\section{Gelenknahe Frakturen der oberen Extremität im Kindes- und Jugendalter}

\section{Worauf ist zu achten?}

In der Kinder- und Jugendtraumatologie wird den knöchernen Verletzungen in der Nähe der Wachstumsfugen aus zweierlei Gründen besonderes Augenmerk geschenkt: Zum einen stellt die Wachstumsfuge mit dem ihr innewohnenden Längenwachstum und damit Korrekturpotenzial nach einer Frakturheilung in Fehlstellung eine indikatorisch wichtige Größe bei der Wahl des therapeutischen Verfahrens dar. Zum anderen kann es bei einer Schädigung der Wachstumsfuge im Verlauf der Behandlung zu posttraumatischen Fehlstellungen kommen, weshalb nach wie vor bei einer derartigen Verletzung die Maxime der möglichst anatomischen Rekonstruktion der Wachstumsfuge - analog zur Gelenkfläche beim Erwachsenen - gilt.

Vor allem nach Verletzungen in der Region des Ellenbogens mit seinen nur wenig am Längenwachstum beteiligten Wachstumsfugen ist es heute bei den meisten Behandlern aufgrund zahlreicher Publikationen und eigener Erfahrung bekannt, dass selbst geringe Fehlstellungen, v. a. in der Frontalebene, gar nicht oder nur minimal durch das Längenwachstum korrigiert werden können (z. B. der Cubitus varus nach suprakondylärer Humerusfraktur) und daher einer operativen Therapie bedürfen $[2,3]$.

Allerdings beobachten wir in umgekehrter Weise zunehmend, dass nach Verletzungen in der Nähe stark wach- sender Fugen (distaler Radius und proximaler Humerus) in der Kinder- und Jugendtraumatologie teilweise von einem zu hohen Korrekturpotenzial ausgegangen wird. Dies gilt v. a. ab dem Adoleszentenalter, wenn die Wachstumsfuge aufgrund des weitgehend abgeschlossenen Längenwachstums nur noch ein geringes Korrekturpotenzial besitzt.

Im vorliegenden Beitrag wird zunächst auf die klassischen Verletzungen der Ellenbogenregion mit ihren Operationsindikationen eingegangen. Darüber hinaus werden bezüglich der eigentlich weniger kritischen Regionen des Handgelenks und des Schultergelenks die Situationen, Alters- und Korrekturgrenzen besprochen, innerhalb derer sich die konservative Therapie nach einer knöchernen Verletzung unserer Ansicht nach bewegen sollte.

\section{Radiologie}

Neben der falschen Einschätzung des Korrekturpotenzials ist die Beurteilung von Röntgenbildern der Ellenbogenregion beim Kind ein weiterer Faktor, der zu der im Vergleich zum Erwachsenen deutlich höheren Anzahl von Schlichtungsverfahren führt.

Grundsätzlich gilt, dass die Anfertigung der beiden Standardprojektionen in der Frontal- und Sagittalebene des Ellenbogens für den Geübten nahezu immer die Diagnosestellung ermöglicht. Suchende Röntgenaufnahmen und das Röntgen der unverletzten Gegenseite zum Vergleich sind zu vermeiden. Zielaufnahmen können für die Diagnose einer Radiushalsfraktur im Einzelfall hilfreich sein.

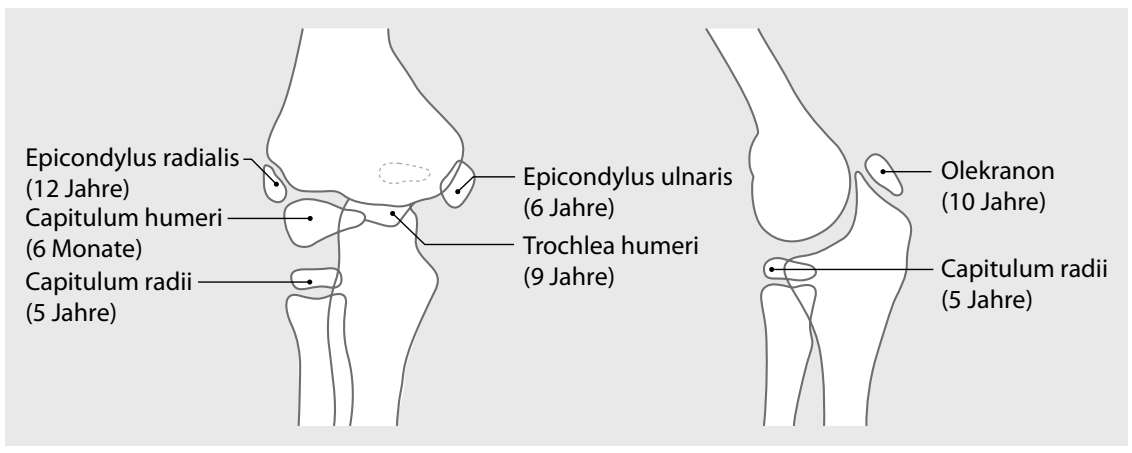

Abb. $1 \Delta$ Zeitliches Auftreten der Ossifikationskerne des Ellenbogens im Kindesalter im Röntgenbild 


\section{Kindliches Trauma}
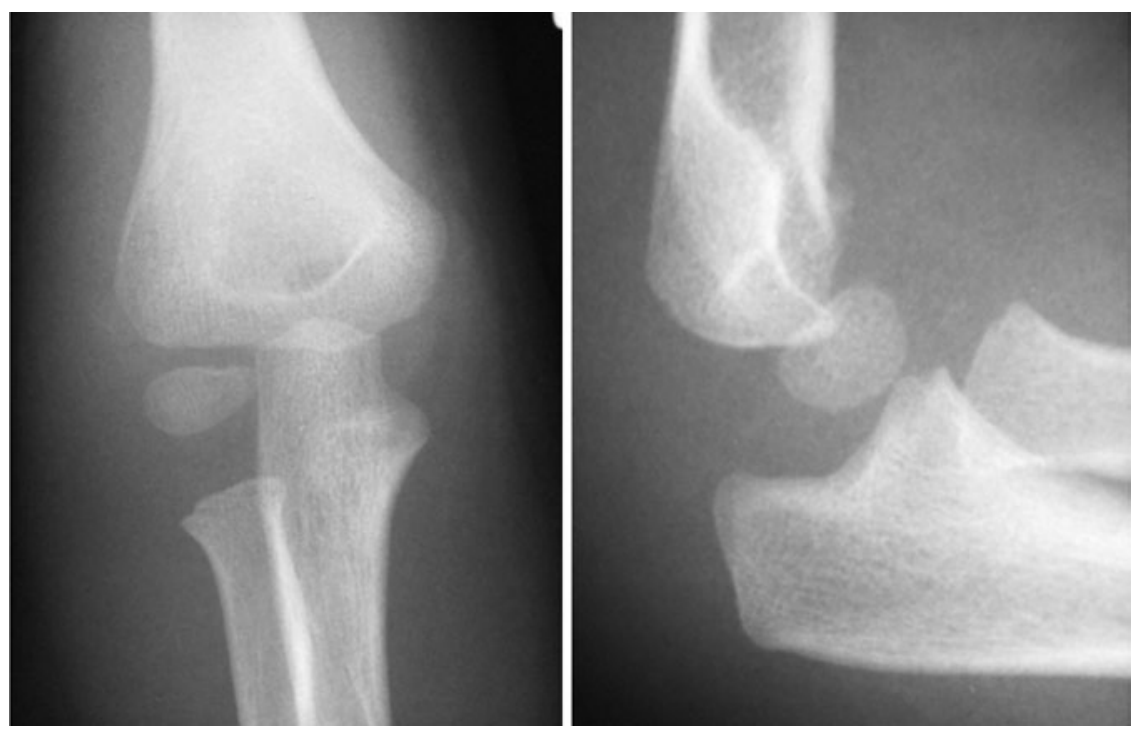

Abb. 24 Unfallaufnahme einer Condylus-radialis-Fraktur eines 5-jährigen Jungen, Fraktur nicht disloziert und daher schwer diagnostizierbar
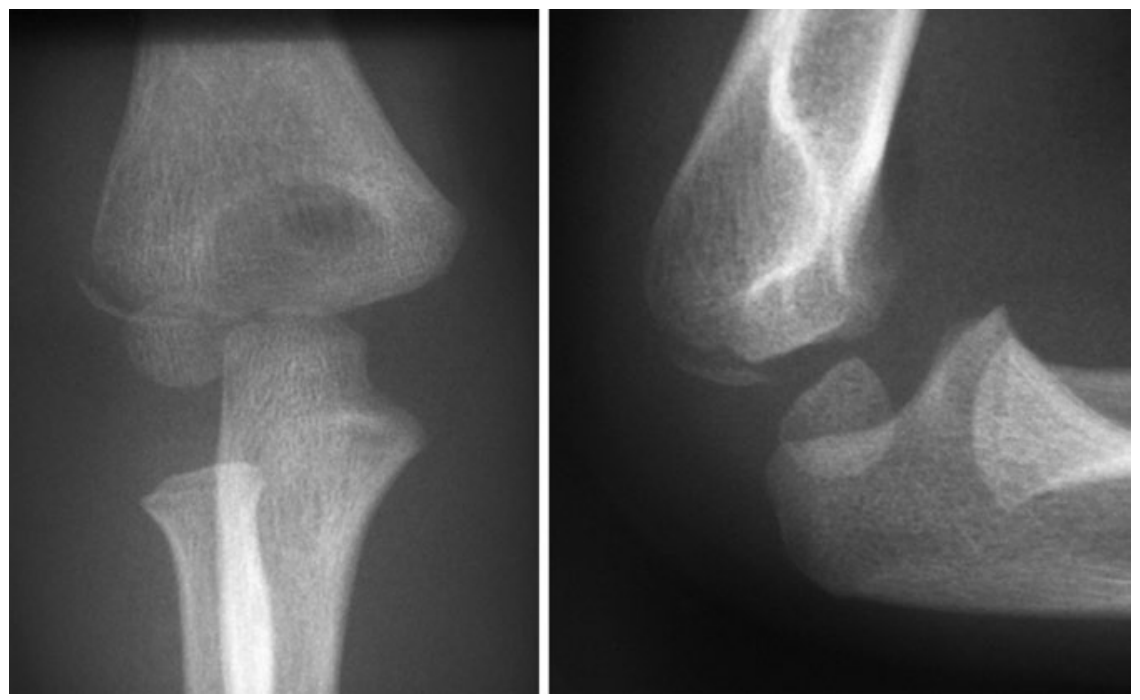

Abb. $3<$ Sekundärröntgenbefund einer Condylus-radialis-Fraktur 5 Tage nach initialer Diagnostik und zwischenzeitlicher Ruhigstellung, Fraktur nun sekundär disloziert und deutlich sichtbar
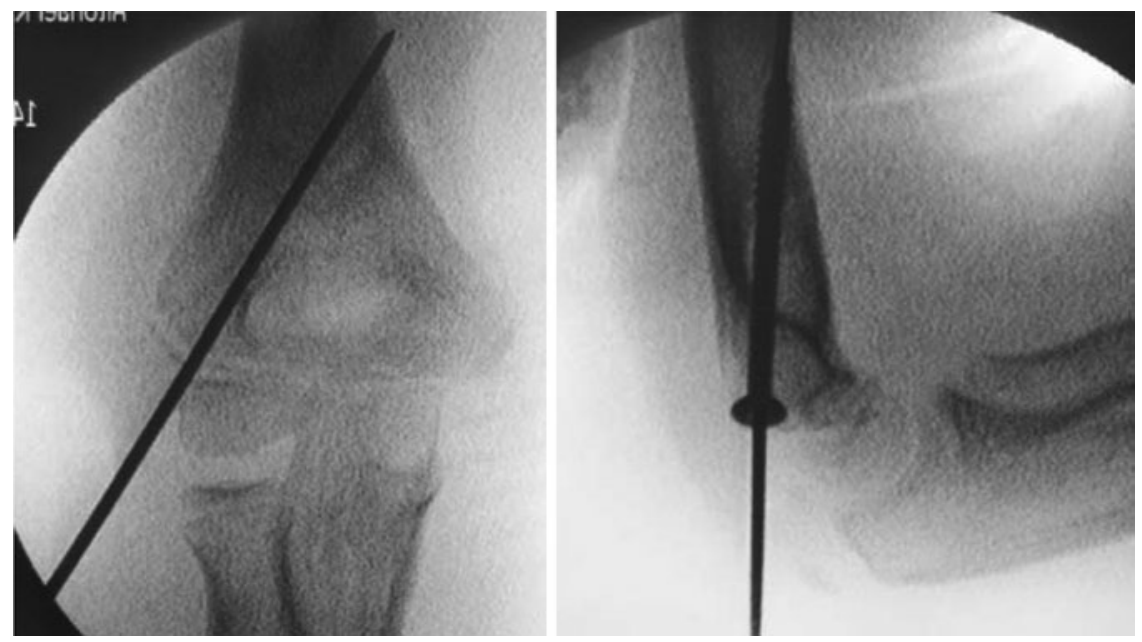

Abb. $4<$ Kompressionsschraubenosteosynthese bei dislozierter Fraktur mittels kanülierter 4-mm-Schraube, intraoperative Durchleuchtung 
Da je nach Alter verschiedene Ossifikationskerne den kindertraumatologisch weniger geschulten Behandler verwirren können, empfiehlt sich hier die Zuhilfenahme eines einschlägigen Röntgenatlas der Kinderradiologie (• Abb. 1, [1]).

\section{Klassische Ellenbogenverletzungen}

Im Folgenden werden die 3 klassischen Probleme am Ellenbogen des Kindes nach Trauma (es existieren weitere) besprochen.

\section{Frakturen des Condylus radialis}

Bei entsprechender Klinik (Druckschmerz lateral über dem distalen Humerus) erfolgt die Standardröntgendiagnostik, wobei die Condylus-radialis-Fraktur selbst für den erfahrenen Behandler im initialen Röntgenbild oft nur schwer zu diagnostizieren ist ( $\bullet$ Abb. 2).

Stabile Frakturen können konservativ durch Ruhigstellung im Oberarmcast ausbehandelt werden. Instabile Frakturen werden durch Kompressionsschraubenosteosynthese versorgt. Die Unterscheidung zwischen diesen beiden Entitäten kann nicht immer primär, d. h. am Unfalltag erfolgen, ohne einen unverhältnismäßig hohen Aufwand, z. B. durch eine MRT (Magnetresonanztomographie), zu betreiben.

Es ist legitim, bei allen unklaren Befunden eine symptomatische Ruhigstellung in der Oberarmcastschiene durchzuführen und nach 5 Tagen erneut zu röntgen (- Abb. 3). Hat sich die Fraktur zu diesem Zeitpunkt durch den Muskelzug der Finger- und Handgelenkstrecker sekundär disloziert, ist ebenfalls die Kompressionsschraubenosteosynthese indiziert (• Abb.4). Bei weiterhin unverschobener Fraktur kann konservativ weiterbehandelt werden, wobei nach weiteren 5 Tagen eine sekundäre Dislokation röntgenologisch sicher ausgeschlossen werden sollte.

Bei übersehener Fraktur kann es zur Ausbildung einer Pseudarthrose mit sog. Fischschwanzdeformität und konsekutiver dauerhafter Funktionseinschränkung kommen (• Abb. 5).

Trauma Berufskrankh 2013 · 15[Suppl 1]:59-66 DOI 10.1007/s10039-012-1916-3

(c) Springer-Verlag Berlin Heidelberg 2013

\section{D.W. Sommerfeldt \\ Gelenknahe Frakturen der oberen Extremität im Kindes- und Jugendalter. Worauf ist zu achten?}

\section{Zusammenfassung}

Frakturen im Bereich des Ellenbogens im Kindes- und Jugendalter lassen sich bei ausreichender Erfahrung meist anhand der beiden Standardprojektionen in der Frontal- und Sagittalebene diagnostizieren. Die Condylus-radialis-Fraktur ist im initialen Röntgenbild oft nur schwer zu erkennen. In solchen Fällen erfolgt bis zur erneuten radiologischen Diagnostik nach 5 Tagen eine symptomatische Ruhigstellung. Stabile, nichtdislozierte Condylus-radialis-Frakturen können durch Ruhigstellung im Oberarmcast behandelt werden, bei instabilen Frakturen ist eine Kompressionsschraubenosteosynthese indiziert. Die Monteggia-Verletzung muss innerhalb eines kleinen Zeitfensters korrekt diagnostiziert und therapiert werden, um aufwendige Rekonstruktionen zu vermeiden. Bei suprakondylärer Humerusfraktur mit Fehlstellung in der Frontalebene erfolgt in der Regel eine Reposition mit Osteosynthese. Die Wachstumsfugen im Bereich von Schulter und Handgelenk weisen im Bereich der oberen Extremität das höchste Wachstums- und Korrekturpotenzial auf, dennoch gibt es hier auch Situationen, bei denen die Grenzen dieser Korrekturfähigkeit erreicht werden und ein Eingriff in Narkose indiziert ist.

\section{Schlüsselwörter}

Ellenbogen · Schulter $\cdot$ Handgelenk . Wachstumsfuge · Korrekturpotenzial

\section{Fractures close to the joints of the upper extremities in childhood and adolescence. What is important?}

\section{Abstract}

With sufficient experience fractures in the region of the elbow during childhood and adolescence can be diagnosed with conventional X-rays in the frontal and sagittal planes. Radial condyle fractures can be difficult to diagnose in initial X-ray images and in such cases symptomatic immobilization should be initiated and control $\mathrm{X}$-rays taken 5 days later. If stable, non-dislocated radial condyle fractures can be treated by immobilization in an upper arm cast. For unstable fractures compression screw osteosynthesis is indicated. Monteggia fractures must be correctly diagnosed and treated within short time frame in order to avoid extensive reconstruction pro- cedures. For supracondylar humeral fractures with malpositioning in the frontal plane, reduction and osteosynthesis are carried out. Growth plates close to shoulder and wrist joint have the highest growth and correction potential within the region of the upper extremity; nevertheless, situations can occur where the limits of this corrective potential are reached and surgical intervention under anesthesia is indicated.

\section{Keywords}

Elbow joint $\cdot$ Shoulder $\cdot$ Wrist $\cdot$ Epiphyseal plates . Correction potential

\section{Monteggia-Verletzung}

Über die akute oder übersehene (sog. „missed Monteggia“) Monteggia-Verletzung, also die Ulnaschaftfraktur mit Luxation des Radiusköpfchens, oder die Äquivalentverletzungen mit ulnarer, meist proximaler Biegungs- oder Olekranonfraktur, wird häufig und überproportional viel publiziert $[7,8]$. Dennoch handelt es sich dabei nach wie vor um eine der häufigeren Verletzungen im Kindesalter, bei denen ein Schlichtungsverfahren zugunsten des Patienten entschieden wird. Dies hat sicherlich mehrere Gründe:
1. Die Verletzung ist akut nicht immer eindeutig im Röntgenbild diagnostizierbar, und das Radiusköpfchen steht oft zunächst subluxiert, um dann bis zur nächsten Röntgenkontrolle vollständig zu luxieren.

2. Die Monteggia-Verletzung bleibt eine seltene Verletzung, auch in kindertraumatologischen Zentren.

3. Die Behandlung muss innerhalb eines kleinen Zeitfensters korrekt diagnostiziert und therapiert werden, um aufwendige Rekonstruktionen zu vermeiden. 

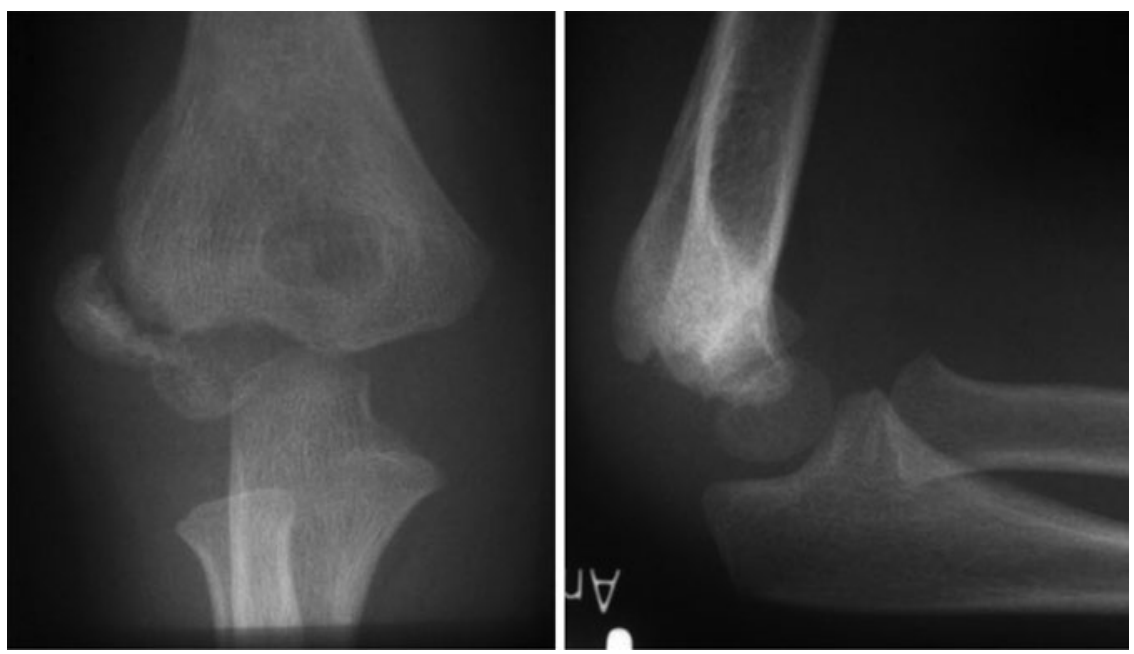

Abb. $5 \Delta$ Ausbildung einer Pseudarthrose mit sog. Fischschwanzdeformität und konsekutiver dauerhafter Funktionseinschränkung bei initial übersehender Condylus-radialis-Fraktur
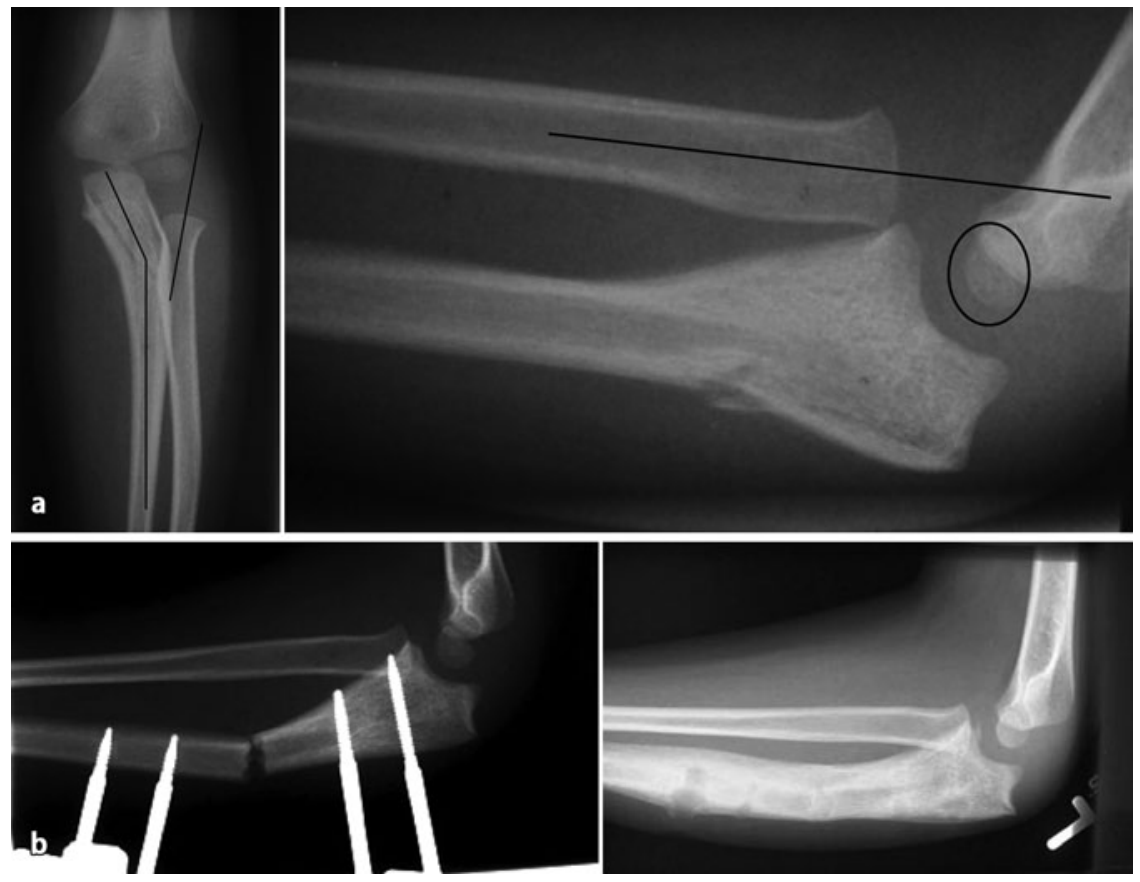

Abb. $6 \Delta$ Monteggia-Verletzung, a Unfallaufnahmen mit klar erkennbarer und dennoch übersehener Monteggia-Verletzung bei einem 4-jährigen Jungen, $\mathbf{b}$ nach verspäteter Diagnosestellung mit bereits eingetretener relativer Radiusverlängerung nach Korrekturosteotomie und Fixateur-externe-Anlage. (Mit freundl. Genehmigung von Thieme, aus [5])

4. Die Verletzung wird oft als Chassaignac-Läsion bagatellisiert und nicht korrekt therapiert.

5. Häufig wird lediglich die proximale Ulnafraktur diagnostiziert.

Bei der akuten Verletzung liegt, anders als bei der Chassaignac-Läsion, nahezu immer eine ausgeprägte Schwellung der proximalen Ulnaregion vor, auch wenn die Fraktur inkomplett ist. Bei vollständig nach beugeseitig luxiertem Radiusköpfchen ist dieses in der Ellenbeuge tastbar. Die Akutdiagnostik zeigt im Idealfall die für die Radiusköpfchenluxation verantwortliche Ulnaverletzung und die nicht mehr auf das Capitulum radii projizierbare Radiushalssachse (• Abb. 6a).

Die Therapie besteht in der korrekten, sofortigen Diagnose mit anschließender Reposition und Stabilisierung der Ulnaoder Olekranonfraktur. Durch die Re- position und die wiederhergestellte Spannung der Membrana interossea reponiert sich das Radiusköpfchen intraoperativ spontan in das proximale Radioulnargelenk. Nur in den seltenen Fällen eines eingeschlagenen Lig. anulare muss offen reponiert werden.

Bei den verspätet, aber innerhalb eines Jahres diagnostizierten Verletzungen kann in der Regel durch eine aufwendige Korrekturosteotomie der Ulna noch eine Kongruenz der Gelenkflächen erreicht werden, auch wenn bereits eine relative Radiusverlängerung eingetreten sein sollte (- Abb. 6b). Ist das Radiusköpfchen jedoch bereits verformt und/oder ist die Fossa der radialseitigen Ulna durch das permanente Remodelling des Knochens verschwunden, ist diese posttraumatische Deformität in der Regel nicht mehr korrigierbar [6]. Bei Schmerzen und einer Funktionseinschränkung der Flexion bleibt in diesen Fällen oft nur noch die symptomatische Resektion des Radiusköpfchens nach Abschluss des Längenwachstums.

\section{Rotationsfehler nach suprakondylärer Humerusfraktur}

Generell gilt für den distalen Humerus, dass das Potenzial der dortigen Wachstumsfuge zur Korrektur von Fehlstellungen eng begrenzt ist. Zudem können Fehlstellungen in der Hauptbewegungsebene, also der Sagittalebene, besser korrigiert werden als diejenigen mit einer Lokalisation in der Frontalebene oder anders gesagt: Eine Antekurvationsfehlstellung des distalen Humerus korrigiert besser als ein Cubitus varus.

Dies bedeutet, dass bei suprakondylärer Humerusfraktur mit Fehlstellung in der Frontalebene in der Regel eine Reposition mit/oder ohne Osteosynthese erfolgt. Das Vorliegen eines Rotationsfehlers am distalen Humerus begünstigt die Entstehung einer solchen Fehlstellung, da bei dann deutlich reduziertem Fragmentkontakt die Instabilität größer und dadurch die primäre oder sekundäre Dislokation deutlich wahrscheinlicher werden. Der Nachweis eines Rotationsfehlers ist demzufolge ein Gradmesser für die Instabilität dieser Verletzung und weist bereits 

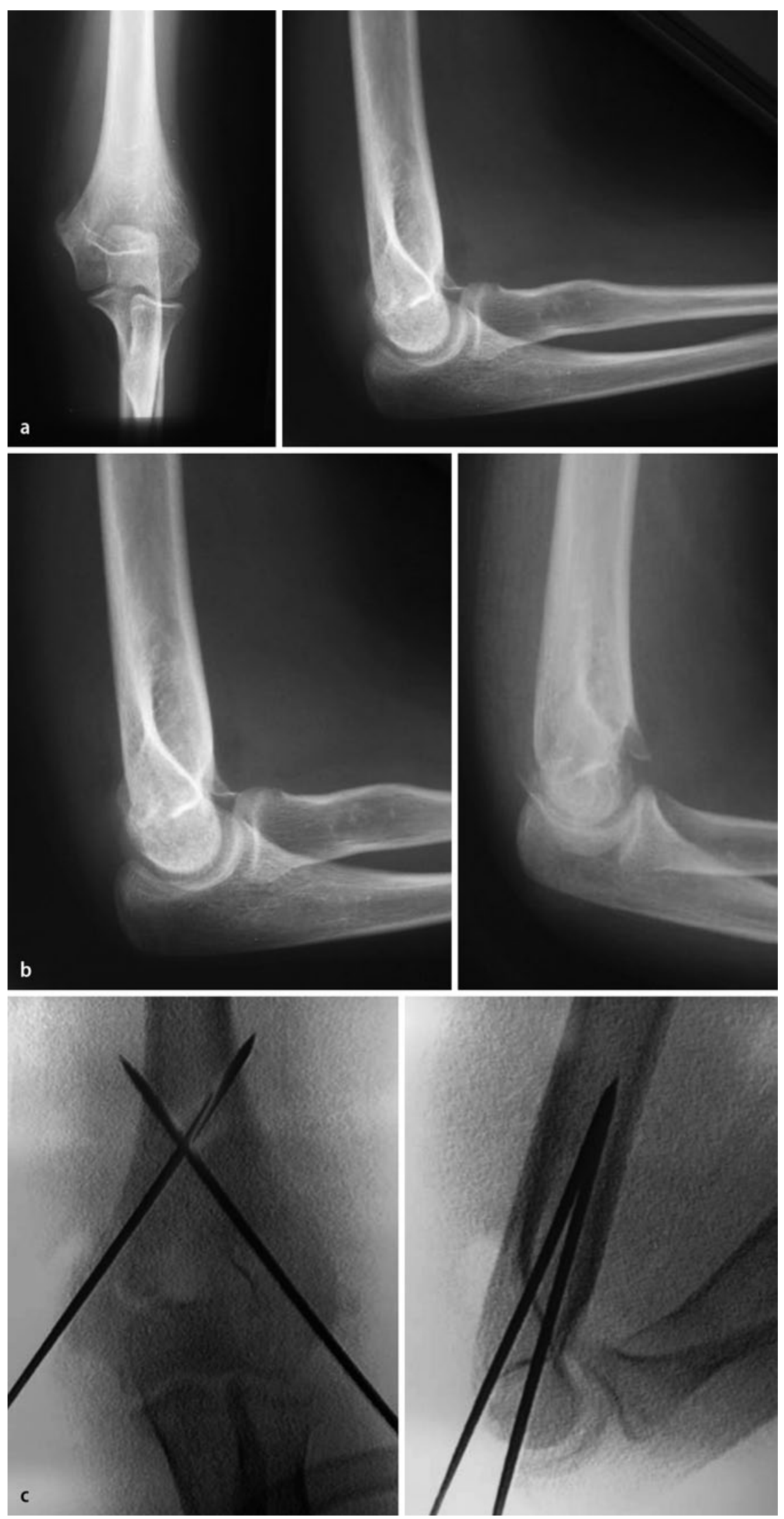

primär auf die Gefahr einer sekundären Dislokation hin.

Bezüglich der Erkennung eines solchen Rotationsfehlers in konventionellen Röntgenaufnahmen sind die Begriffe Rotationssporn, Kalibersprung bzw. Rotationsfehlerquotient von Bedeutung [3]: In dem in $\mathbf{A b b} . \mathbf{7}$ dargestellten Fall lag eine suprakondyläre Humerusfraktur bei einer adoleszenten Patientin nach bereits erfolgtem Fugenschluss vor. In den initialen Röntgenaufnahmen ( $\mathbf{\bullet}$ Abb. 7a) war eine durch die Hyperextension beim Trauma entstandene Antekurvationsfehlstellung mit aufgehobenem EpiphysenDiaphysen-Winkel darstellbar. Beugeseitig fand sich eine Kortikalisschuppe, die oft fälschlicherweise als Rotationssporn bezeichnet wird. Ein Rotationsfehler lag jedoch in diesem Fall - noch - gar nicht vor, die beiden Hauptfragmente zeigten vollständigen Knochenkontakt, der Rotationsfehlerquotient hatte den Wert 0 . Erst in der Röntgenkontrolle nach einigen $\mathrm{Ta}$ gen begann die Fraktur, ausgelöst durch den Muskelzug trotz Oberarmcast, auch in der Frontalebene sekundär zu dislozieren, was sich in der seitlichen Röntgenaufnahme im Vergleich zur Unfallaufnahme am nun vorhandenen Rotationssporn mit Kalibersprung und somit positivem Rotationsfehlerquotienten zeigte (• Abb. 7b). Aus unserer Sicht hätte bereits am Unfalltag aufgrund der Antekurvationsfehlstellung und des bereits abgeschlossenen Längenwachstums eine Indikation zur Reposition und Osteosynthese bestanden, welche nach der Kontrolluntersuchung durch den sekundären Rotationsfehler bei instabiler Fraktur erhärtet wurde. $\square$ Abb. 7c zeigt die intraoperativen Röntgenkontrollaufnahmen mit:

Abb. 74 Suprakondyläre Humerusfraktur einer adoleszenten Patientin nach bereits erfolgtem Fugenschluss, a initiale Röntgendiagnostik, b Sagittalaufnahmen des Ellenbogens, links Hyperextensionsfehlstellung noch ohne, rechts 5 Tage später nun auch mit sekundärer Dislokation in der Frontalaufnahme und sichtbarem Rotationssporn, c intraoperative Durchleuchtung nach KirschnerDraht-Osteosynthese 

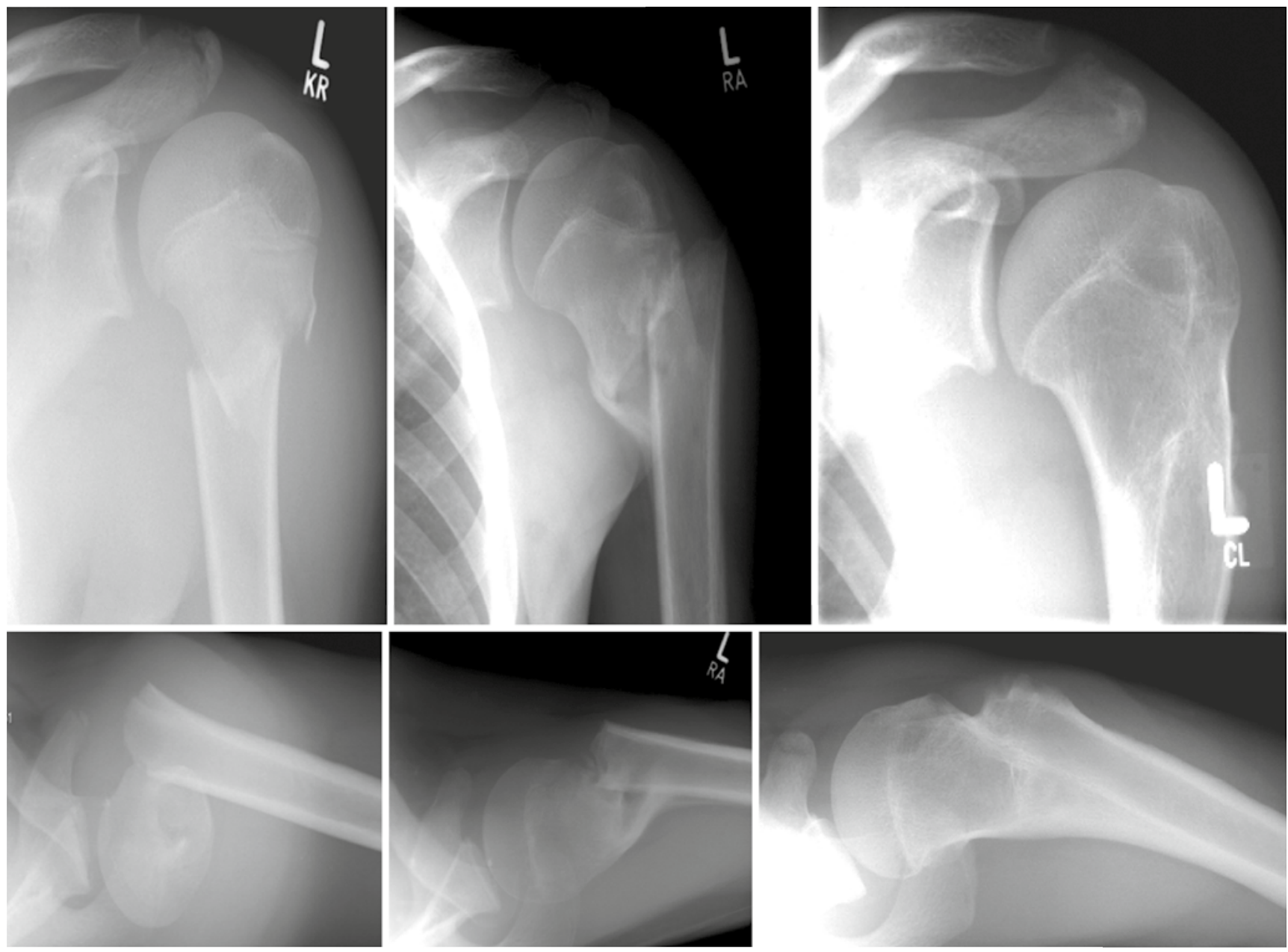

Abb. $8 \Delta$ Subkapitale Humerusfraktur, Verlauf nach konservativer Therapie bei Schädel-Hirn-Trauma Grad III bei einem 14-jährigen Jungen. (Aus [2])

- wiederhergestelltem Epiphysen-Diaphysen-Winkel und

- nicht mehr vorhandenem Rotationsfehler ohne Kalibersprung.

\section{Operationsindikation im Schulter- und Handgelenkbereich}

Im Bereich der oberen Extremität weisen Schulter und Handgelenk das höchsten Wachstums- und Korrekturpotenzial auf. Im Folgenden soll auf diejenigen Situationen fokussiert werden, bei denen die Grenzen dieser Korrekturfähigkeit erreicht werden und in der Regel ein Eingriff in Narkose indiziert ist.

\section{Subkapitale Humerusfraktur}

Sie ereignet sich meist durch Sturz vom Fahrrad oder Pferd auf die Schulter oder beim Sport im direkten Gegnerkontakt. Betroffen sind überwiegend ältere Kinder und Jugendliche ab dem 10. Lebensjahr.

Generell verfügt die proximale Wachstumsfuge des Humerus mit etwa $80 \%$ Anteil am Längenwachstum über ein hohes Korrekturpotenzial bezüglich Fehlstellungen. Sofern noch mindestens 2 Jahre Restwachstum vorhanden sind, gilt diese Fraktur, auch bei höhergradigen Fehlstellungen, als Domäne der konservativen Therapie mit symptomatischer Ruhigstellung für etwa 2 Wochen und anschließender frühfunktioneller Therapie.

Allerdings sind häufig Patienten betroffen, die kurz vor dem Wachstumsabschluss stehen oder diesen bereits erreicht haben. In diesen Fällen kann mittels konservativer Therapie zwar häufig eine Bruchheilung erreicht werden; die verbliebene Fehlstellung korrigiert sich jedoch nicht mehr zuverlässig ([2], - Abb. 8).

Aufgrund des hohen Freiheitsgrades des Schultergelenks fallen Funktionseinschränkungen im weiteren Verlauf oft erst bei extremen Belastungen oder Jahre nach dem Unfall auf.

Da mit der elastisch-stabilen intramedullären Nagelung (ESIN) ein seit Jahren eingeführtes, zuverlässiges und minimalinvasives Verfahren zur Stabilisierung zur Verfügung steht, das zudem im Gegensatz zu der früher angewandten perkutanen Kirschner-Draht-Osteosynthese keine additive Ruhigstellung erfordert und somit eine frühfunktionelle Nachbehandlung erlaubt, halten wir bei allen adoleszenten Patienten mit kurz vor Wachstumsabschluss stehenden oder bereits verschlossenen Fugen bei einer Fehlstellung von über $20^{\circ}$ Varus- oder Ante-/Rekurvationsfehlstellung und über $10^{\circ}$ Valgusfehl- 


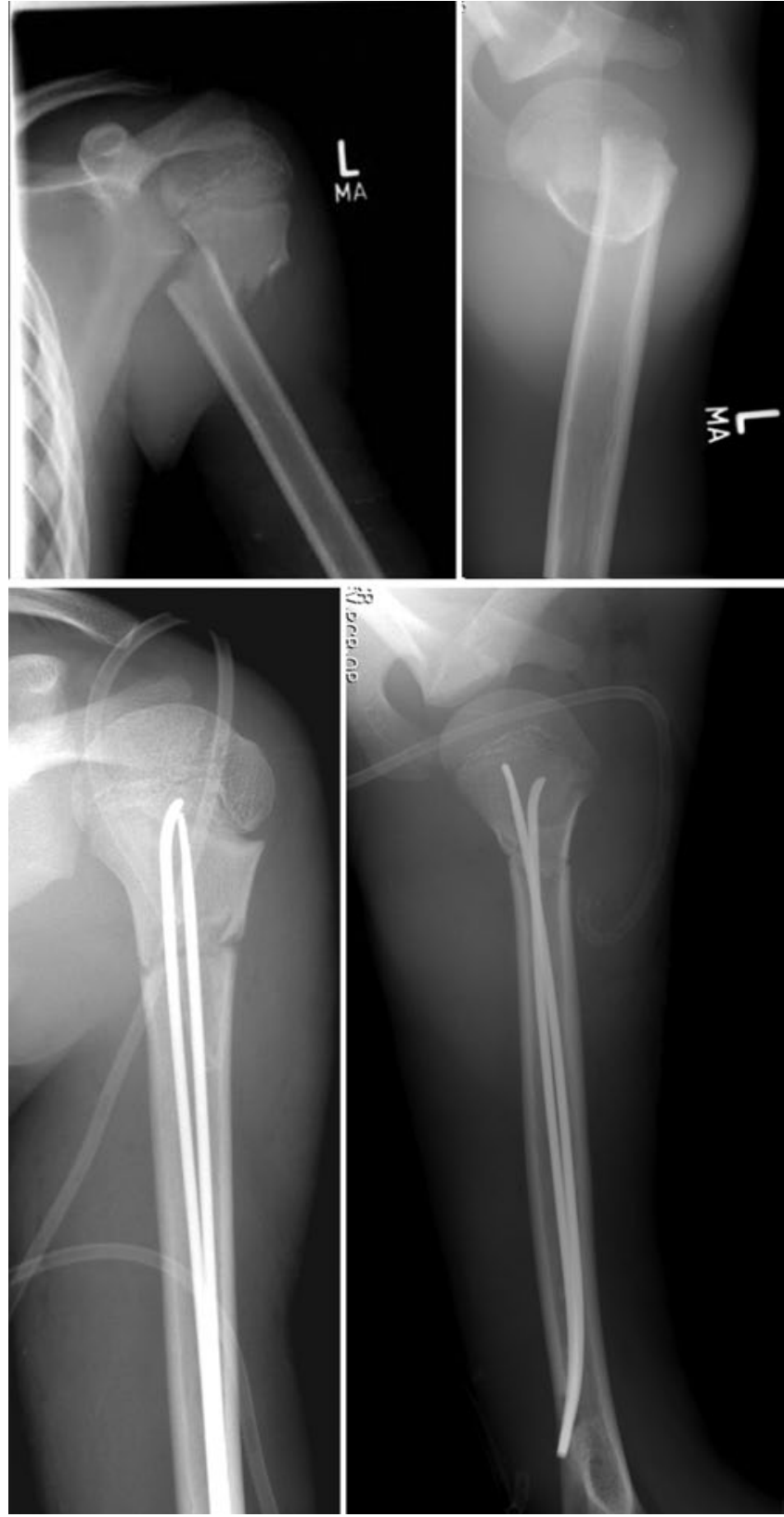

Abb. $9<$ Intramedulläre Schienung (elastisch-stabile intramedulläre Nagelung: ESIN) einer subkapitalen Humerusfraktur bei einem 11-Jährigen. (Aus [2])

stellung und/oder Ad-latus-Dislokation über halbe Schaftbreite eine intramedulläre Schienung für indiziert ([2], - Abb. 9).

\section{Distale, metaphysäre Radiusfraktur}

Ähnlich wie bei der subkapitalen Humerusfraktur gilt auch für diese Frakturzone, dass aufgrund des starken Längenwachstums der Wachstumsfuge im Kindesalter ein erstaunlich hohes sekundäres Korrekturpotenzial für Fehlstellungen v. a. in der sagittalen Hauptbewegungsebene besteht. Hier können sich bis zum 10. Lebensjahr Achsfehlstellung bis $30^{\circ}$ in der Sagittal- faktor nicht mehr in ausreichender Weise zur Aufrichtung der Fehlstellung beitragen kann, müssen alters- und situationsangepasste, operative Maßnahmen an die Stelle der konservativen Therapie treten [4].

Wir empfehlen ab dem 10. Lebensjahr bei Fehlstellungen über $20^{\circ}$ in der Sagittalebene und über $10^{\circ}$ in der Frontalebene deren Reposition mit intraoperativer Entscheidung über die Notwendigkeit einer Osteosynthese. Für Patienten nahe am Wachstumsabschluss sollten in allen Ebenen Achsfehlstellungen nur noch bis maximal $10^{\circ}$ toleriert werden. Nach dem Fugenschluss gelten im Wesentlichen die Therapierichtlinien der Erwachsenentraumatologie [2]. In jedem Fall gilt, dass nach einem Eingriff in Narkose eine vollständige Reposition der Achsfehlstellung mit stabiler Retention zu fordern ist

(- Abb. 10). Keinesfalls sollten Mehrfacheingriffe bzw. Nachrepositionen erforderlich werden [3].

\section{Diskussion}

Die Kinder- und Jugendtraumatologie weist im Bereich der oberen Extremität eine gewisse, in jedem Fall aber endliche Anzahl von für das Wachstumsalter spezifischen Verletzungsformen auf. Da einige davon unerkannt oder inadäquat behandelt zu dauerhaften Funktionseinschränkungen oder aufwendigen Korrektureingriffen mit nicht immer guten Ergebnissen führen können, ist eine Kenntnis dieser Verletzungen für jeden unfallchirurgisch tätigen Arzt von Bedeutung. Dies gilt in besonderer Weise für die Region des Ellenbogens, dessen angrenzende Wachstumsfugen verbliebene Achsabweichungen nur in sehr begrenztem Maß korrigieren können.

Gleichzeitig kommen aber auch immer wieder in den Regionen mit eigentlich hoher Korrekturfähigkeit unbefriedigende Ergebnisse vor. Dies ist in der Fehleranalyse dann nicht selten die Folge eines zu optimistisch eingeschätzten Korrekturpotenzials der angrenzenden Wachstumsfuge oder einer für den individuellen Patienten nicht geeigneten Therapie. In der Konsequenz darf dies jedoch nicht zu einem unfallchirurgischen Aktionismus mit breiter Indikationsstellung 


\section{Kindliches Trauma}
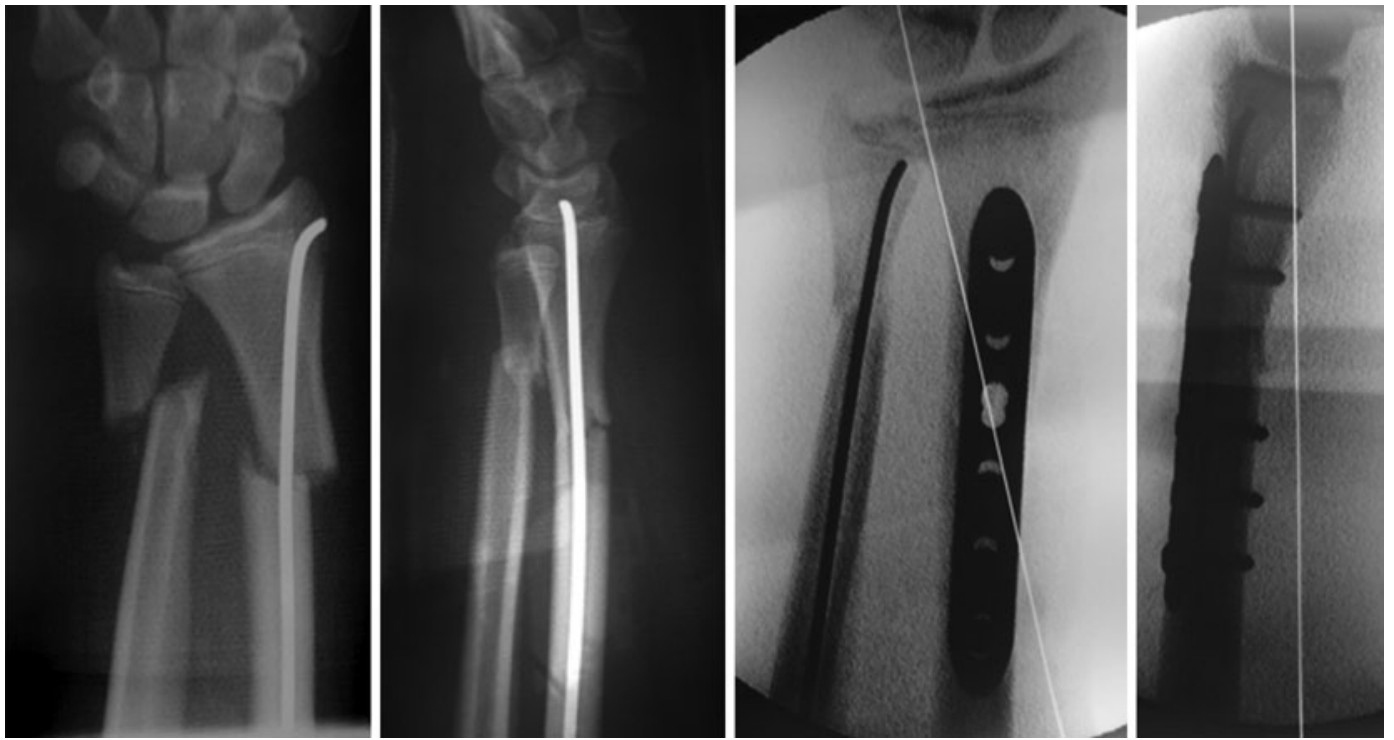

Abb. $10 \varangle$ Zunächst insuffiziente, da instabile Osteosynthese einer distalen, metaphysären Unterarmfraktur mittels isolierter ESIN (elastisch-stabile intramedulläre Nagelung) des Radius bei einem 15-Jährigen mit Umstieg auf Plattenosteosynthese des Radius und ESIN der Ulna am Folgetag

für operative Maßnahmen über alle $\mathrm{Al}$ tersgruppen hinweg führen. Idealerweise ist vor jeder Therapieentscheidung eine genaue und individuelle Analyse des Entwicklungsstadiums des jeweiligen Patienten inklusive Bestimmung des Skelettalters mit Wachstumsprognose $\mathrm{zu}$ fordern. Daraus ergibt sich in den allermeisten Fällen sehr logisch die Entscheidung für die richtige Therapiemaßnahme.

Nur in seltenen Fällen, z. B. bei der pulslosen suprakondylären Humerusfraktur, besteht die Notwendigkeit zum sofortigen Handeln. In vielen Fällen kann - gerade im Zeitalter des schnellen Datentransfers - eine Zweitmeinung durch den kindertraumatologisch erfahreneren Kollegen eingeholt werden, ohne durch den Zeitverlust einen Behandlungsnachteil für den betroffenen Patienten in Kauf nehmen zu müssen.

\section{Korrespondenzadresse}

\section{PD Dr. D.W. Sommerfeldt}

Abteilung für Kinder- und Jugendtraumatologie, Altonaer Kinderkrankenhaus gGmbH, Bleickenallee 38, 22763 Hamburg dirk.sommerfeldt@kinderkrankenhaus.net

Interessenkonflikt. Der korrespondierende Autor gibt an, dass kein Interessenkonflikt besteht.

The supplement containing this article is not sponsored by industry.

\section{Literatur}

1. Brossmann J, Freyschmidt J, Czerny C et al (2000) Grenzen des Normalen und Anfänge des Pathologischen in der Radiologie des kindlichen und erwachsenen Skeletts. Thieme, Stuttgart New York

2. Dietz H-G, Illing P, Schmittenbecher P et al (2011) Praxis der Kinder- und Jugendtraumatologie. Springer, Berlin Heidelberg New York

3. Laer L von, Kraus R, Linhart W (2007) Frakturen und Luxationen im Wachstumsalter. Thieme, Stuttgart New York

4. Lieber J, Sommerfeldt D (2011) Die diametaphysäre Unterarmfraktur im Kindesalter: Pitfalls und Empfehlungen in der Behandlung, vol 4. Springer, Berlin Heidelberg New York

5. Ruchholtz S, Wirtz D (2010) Orthopädie und Unfallchirurgie essentials. Thieme, Stuttgart New York

6. Slongo T (2008) Korrekturosteotomie der fehlverheilten Monteggia-Verletzung mit einem Fixateur externe. Oper Orthop Traumatol 20:435-449

7. Slongo T, Fernandez F (2011) Die fehlverheilte Monteggia-Verletzung im Kindes- und Jugendalter. Unfallchirurg 4:311-322

8. Vinz H, Festge O, Neu J (2012) Behandlungsfehler bei Armfrakturen im Kindesalter. Trauma Berufskrankh 14(3):388-391 